\title{
PERFORMANCE ASSESSMENT OF INTEGRATED SENSOR ORIENTATION WITH A LOW-COST GNSS RECEIVER
}

\author{
M. Rehak, J. Skaloud \\ École polytechnique fédérale de Lausanne (EPFL), Switzerland - (martin.rehak, jan.skaloud)@epfl.ch
}

KEY WORDS: Sensor orientation, MAV, Lever-arm, Low-cost GNSS

\begin{abstract}
:
Mapping with Micro Aerial Vehicles (MAVs whose weight does not exceed $5 \mathrm{~kg}$ ) is gaining importance in applications such as corridor mapping, road and pipeline inspections, or mapping of large areas with homogeneous surface structure, e.g. forest or agricultural fields. In these challenging scenarios, integrated sensor orientation (ISO) improves effectiveness and accuracy. Furthermore, in block geometry configurations, this mode of operation allows mapping without ground control points (GCPs). Accurate camera positions are traditionally determined by carrier-phase GNSS (Global Navigation Satellite System) positioning. However, such mode of positioning has strong requirements on receiver's and antenna's performance. In this article, we present a mapping project in which we employ a single-frequency, low-cost $(<\$ 100)$ GNSS receiver on a MAV. The performance of the low-cost receiver is assessed by comparing its trajectory with a reference trajectory obtained by a survey-grade, multi-frequency GNSS receiver. In addition, the camera positions derived from these two trajectories are used as observations in bundle adjustment (BA) projects and mapping accuracy is evaluated at check points (ChP). Several BA scenarios are considered with absolute and relative aerial position control. Additionally, the presented experiments show the possibility of BA to determine a camera-antenna spatial offset, so-called lever-arm.
\end{abstract}

\section{INTRODUCTION}

Unmanned Aerial Vehicles (UAVs) are gaining importance in the mapping and monitoring tasks of our environment. The employment of UAVs is attractive in terms of cost, handiness, and flexibility, as they fill a gap between aerial mapping of large areas and local terrestrial surveying. When cm-level accuracy is required for derived geospatial products, the conventional (i.e. indirect) approach of sensor orientation does not deliver satisfactory results unless a large number of GCPs is regularly distributed in the mapped area. This adds a significant cost to a project while becoming impractical in difficult, or inaccessible terrain. For overlapping imagery in block configuration, the need of GCPs can be principally alleviated through precise control of aerial-position (Colomina, 2007).

\subsection{Bundle Adjustment with Aerial Position Control}

ISO benefits from aerial, image, and possible ground data. It is a robust and efficient method provided proper conditions of sufficient image overlap. Under certain observability conditions, ISO allows system and sensor parameters (e.g. antenna offset, interior camera parameters) to be self-calibrated.

Apart from measurements of tie-features in image and object space, the observations and conditions of exterior orientation can be introduced into the adjustment. The number of GCPs can thus be reduced considerably, or under certain configuration may even be completely eliminated. The observation equation that models the relation between the imaging sensor and an antenna-phase centre, for which absolute position is derived, takes the known form:

$$
\mathbf{X}^{m}+\mathbf{v}_{X}^{m}=\mathbf{X}_{0}^{m}+\mathbf{R}_{c}^{m}(\Gamma) \cdot \mathbf{A}^{c}+\mathbf{S}^{m}
$$

where

$\mathbf{X}^{m} \quad$ is the GNSS-derived position for one epoch in a Cartesian mapping frame $m$,

$\mathbf{v}_{X}^{m} \quad$ is the vector of aerial position residuals,

$\mathbf{X}_{0}^{m} \quad$ is the vector of a camera projection centre,

$\mathbf{R}_{c}^{m}(\Gamma) \quad$ is the nine-elements rotation matrix from camera $c$ to $m$ frame parametrised by the traditional Euler angles $\Gamma=(\omega, \varphi, \kappa)$,

$\mathbf{A}^{c} \quad$ is the camera-GNSS antenna lever-arm,

$\mathbf{S}^{m} \quad$ is the possible positioning bias in the GNSS-derived positions.

To reduce the influence of GNSS-positioning bias on aerial positions, temporal differencing of the above equation can be used for two consecutive epochs $t_{i}$ and $t_{j}$, (Li and Stueckmann-Petring, 1992).

$\Delta \mathbf{X}^{m}\left(t_{i j}\right)+\mathbf{v}_{\Delta X}^{m}=\mathbf{X}_{0}^{m}\left(t_{j}\right)-\mathbf{X}_{0}^{m}\left(t_{i}\right)+\left(\mathbf{R}_{c}^{m}\left(\Gamma_{t_{j}}\right)-\mathbf{R}_{c}^{m}\left(\Gamma_{t_{i}}\right)\right) \cdot \mathbf{A}^{c}$

\subsection{Accurate Kinematic GNSS Positioning}

Relatively recent progress in the field of miniaturisation and cost reduction of multi-frequency GNSS receivers and antennae, allowed application of this technology to micro-aerial vehicles (Rehak et al., 2013, Eling et al., 2014), and the creation of the first commercial platforms offering at least accurate aerial position control. With contemporary cutting edge technologies presented on fixed-wing platforms (MAVinci GmbH, 2015, senseFly, 2015) and multirotors (Aibotix GmbH, Part of Hexagon, 2016), the users can benefit from geodetic grade GNSS RTK (Real-Time Kinematic) receivers closely integrated into the platforms processing work-flow as presented, e.g. in the studies of (Survey Group, 2015), or (Gerke and Przybilla, 2016). Although such systems allow in principle accurate aerial position control, this 
capacity is principally dependent on the RTK engine of the embedded multi-frequency and multi-constellation GNSS receiver. The $\mathrm{cm}$-level kinematic satellite positioning is reached through the differential processing of the carrier-phase data. For that purpose, having low noise of phase observations is as important as its continuity (limited number of cycle-slips), a number of measurements and their geometry, a distance to a base etc.; the reason for which manned airborne mapping employs almost exclusively multi-frequency receivers. The ambiguity determination through single-frequency observations is an attractive option as proposed in (Odolinski and Teunissen, 2016) due to an affordability of single-frequency receivers. Furthermore, the proximity to the base (e.g. $<1-2 \mathrm{~km}$ ) theoretically allows, in many MAV missions, single-frequency differential processing. On the other hand, the quality of phase observations is related to the number of aspects that are less favourable in small UAVs: the level of electromagnetic interference, the presence of vibrations and/or high platform dynamics. The receiver build is influential in terms of tracking-loop bandwidth, bit sampling, oscillator stability, and mutlipath reduction. In this respect, the usage of mass-market receivers for precise aerial position control is difficult to generalise and the applicability of a specific setup needs to be evaluated empirically in an operational environment.

Regarding the use, low-cost, single-frequency GNSS receivers are typically employed in automotive industry and consumer electronics. Their use in surveying is possible but often limited to static or low-dynamic applications, e.g. glacier movement or deformation monitoring (Benoit et al., 2015). They have been tested on MAVs in several cases, e.g. in (Stempfhuber and Buchholz, 2011) or (Mongredien et al., 2016). However, their use for the purpose of accurate aerial positioning of aerial imagery in $\mathrm{cm}$ level has not yet been assessed under real mapping conditions and particularly not on fixed-wing platforms. Overall the employment of a single-frequency, low-cost GNSS receiver on MAV platforms is challenging for following reasons:

- Quality of receiver's front-end,

- limited resources for signal sampling/tracking,

- limited band-with/acceleration under which the signal tracking works,

- less channels for signal tracking,

- single-frequency and thus worse capability of resolving ambiguities,

- limited support for synchronisation - input and output timing,

- usually no internal memory,

- lower sampling frequency, i.e. $<10 \mathrm{~Hz}$.

\subsection{Methodology and Paper Structure}

The presented test aims at investigating whether a low-cost $(<$ $\$ 100$ ) mass-market GNSS receiver can provide accurate, i.e. $\mathrm{cm}$ level kinematic positioning, to contribute in ISO. Additionally, the aim is to test the ability to self-calibrate the camera-GNSS antenna lever-arm vector.

Section 2 presents the used equipment, specifically the MAV platform and its sensors. Furthermore, important mission parameters are summarised. The third section concentrates on performance analysis of the low-cost receiver by comparing the camera positions with those calculated from the survey-grade grade receiver. The fourth section is devoted to BA projects with aerial position control with absolute and relative observations derived from the two receivers. Last section draws conclusions from the conducted research work.

\section{EXPERIMENTAL SETUP}

\subsection{Mapping platform}

The drone used for this study is a custom made airplane equipped with the open-source autopilot Pixhawk (Meier et al., 2012). The wingspan is $165 \mathrm{~cm}$ and fuselage length $120 \mathrm{~cm}$. The maximal payload capacity is around $800 \mathrm{~g}$. The operational weight varies between 2200-2800 $\mathrm{g}$ and flying endurance is around 45 minutes. Thanks to is lightweight construction, the launching can be done by hand and it requires only a small place for landing. The platform's main components are depicted in Fig. 1

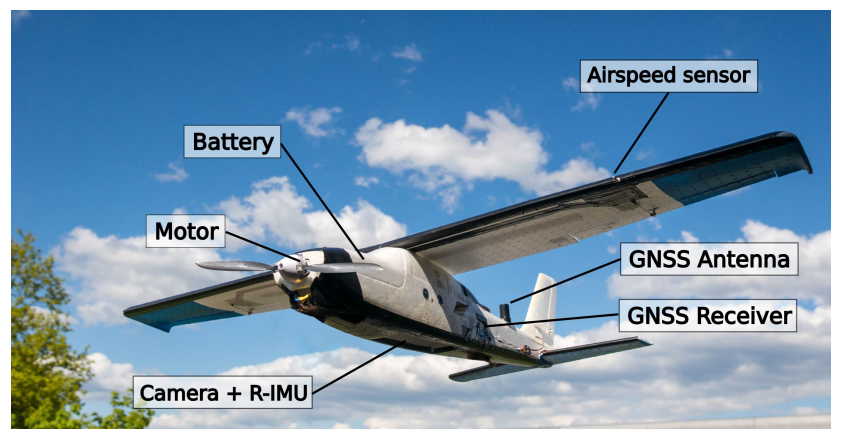

Figure 1. Fixed-wing platform.

\subsection{Sensor Equipment}

The chosen optical sensor is the Sony NEX-5R camera (Sony, 2016). The quality of this mirror-less camera is comparable with a DSLR (digital single-lens reflex) camera despite being considerably smaller (only 111 x 59 x $39 \mathrm{~mm}$ ) and lighter (290 g without lens). These properties make it highly suitable for MAV platforms. The camera is equipped with a $16 \mathrm{~mm}$ fixed Sony lens, which has a reasonable optical quality given its size and weight and offers sufficient stability of the additional distortion parameters thanks to missing optical stabilisation system.

We employ a geodetic grade, multi-frequency, and multi-cons -tellation receiver Javad G3T (Javad GNSS Inc., 2016) and a single-frequency, low-cost GNSS receiver U-Blox NEO-8T (UBlox, 2016). The latter receiver is depicted in Fig. 2. Both receivers are connected via a GNSS signal splitter to a L1/L2 GNSS antenna Maxtenna (Maxtena, 2016).

The advantages of a mass-market over geodetic grade receiver are the price, power consumption, and weight. Tab. 1 summarises the main characteristics of the two employed GNSS receivers. Parameters of the Javad G3T can be modified and purchased upon request. Tab. 1 describes the currently available features. The NEO-8T receiver is mounted on a breakout board (CSG Shop, 2016). The receiver is customised for storing RAW observation on a micro SD card.

\subsection{Test Data}

This study was conducted over agricultural fields and roads. The testing area has a size of approx. 70 ha and is equipped with 25 


\begin{tabular}{|l|c|c|}
\hline Parameter & Javad G3T & U-Blox NEO-8T \\
\hline Size $[\mathrm{mm}]$ & $88 \times 57 \times 12$ & $40 \times 18 \times 10$ \\
\hline Weight $[\mathrm{g}]$ & 47 & 13 \\
\hline Tracking frequencies & $\begin{array}{c}\text { GPS L1/L2/L2C/L5, GLONASS L1/L2, } \\
\text { Galileo E1/E5A, SBAS }\end{array}$ & $\begin{array}{c}\text { GPS L1, GLONASS L1, } \\
\text { BeiDou B1, SBAS }\end{array}$ \\
\hline Tracking channels & 36 per frequency & 72 \\
\hline Rate $[H z]$ & 10 & 5 \\
\hline Built-in RTK & YES & NO \\
\hline Synchronization & PPS + Event & 75 \\
\hline Price (USD) & 10000 & \\
\hline
\end{tabular}

Table 1. Main features of the employed GNSS receivers.

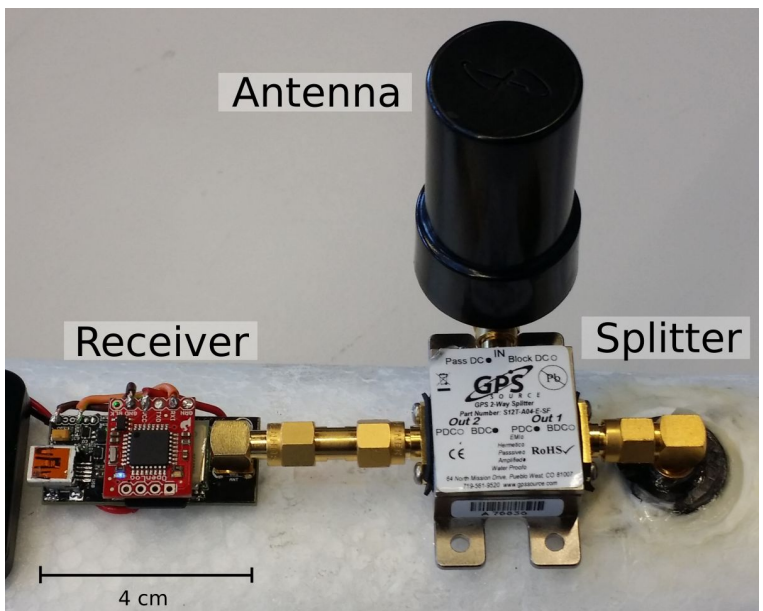

Figure 2. The U-Blox NEO-8T GNSS receiver with a serial data logger, GNSS signal splitter and L1/L2 GNSS antenna Maxtena. During the presented experiment, a second receiver Javad was connected via the splitter to provide a reference trajectory.

permanent markers. The block consists of 12 parallel lines and 4 lines perpendicular to them, flown in two separate flight heights. The data is characterised in Tab. 2. The maximal separation between the MAV and base station was $500 \mathrm{~m}$. The block configuration is depicted in Fig. 3. The ground accuracy was evaluated at independent $\mathrm{ChPs}$.

\begin{tabular}{|l|c|}
\hline Freq. GNSS [Hz] & 10 \\
Flight level [m] & $130 / 160$ \\
Area covered [ha] & 98 \\
Mean GSD [cm] & 4 \\
Overlap [\%] & $80 / 50$ \\
No. of images & 326 \\
No. of tie-points & 6982 \\
No. of GCP/ChP & 23 \\
No. of image obs. & 146694 \\
\hline
\end{tabular}

Table 2. Summary of aquired data.

\section{IN-FLIGHT TRAJECTORY PRECISION}

The GNSS data from both receivers was processed in GrafNav (Novatel, 2016) and interpolated for each camera event. While the reference trajectory has ambiguity fixed throughout the entire flight, the NEO-8T data allows only a float solution. Nevertheless, as long as the float ambiguities converge to a stable value, the float solution may be considered as accurate enough, especially in a relative aerial control.

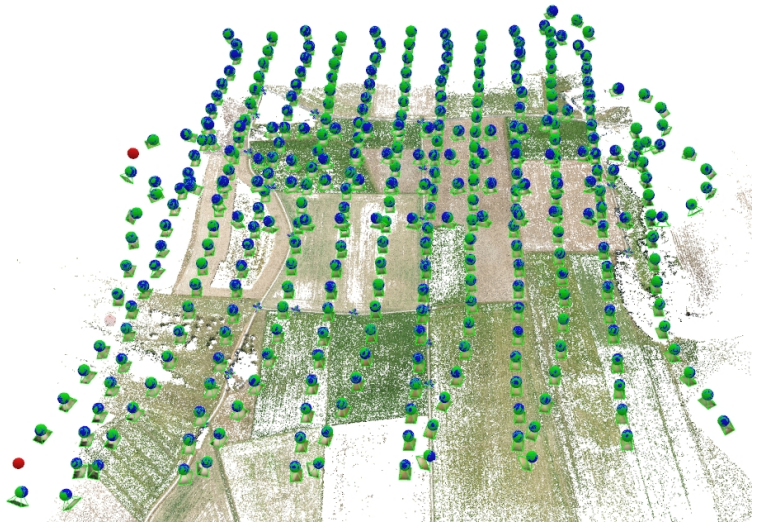

Figure 3. 3D view on the scene with camera stations, GCPs and a point cloud of tie-points from Pix4Dmapper Pro.

The two sets of camera position parameters derived from tested vs. reference data are compared in Fig. 4 in an absolute, and in Fig. 5 in a relative way, i.e. differences between two positions of two consecutive camera stations. It can be seen that rather small $(<10 \mathrm{~cm})$ differences/drift in absolute positions are eliminated by differencing.

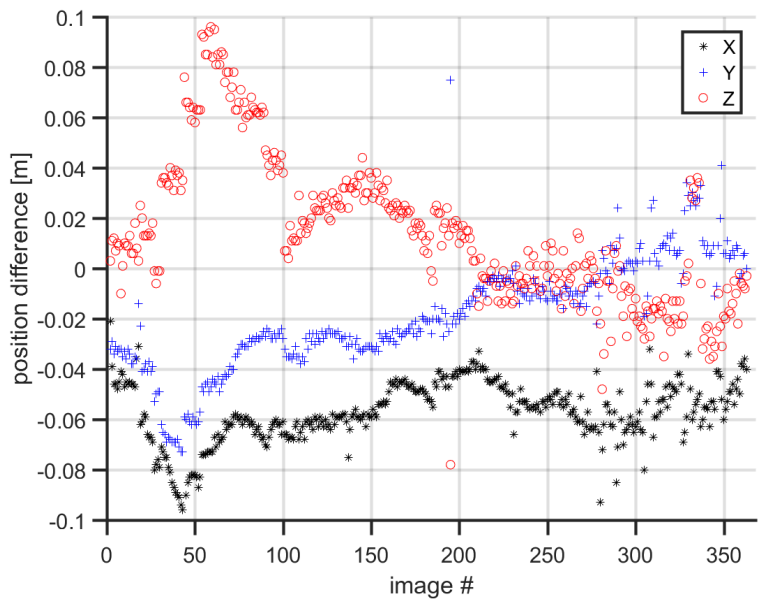

Figure 4. Differences in absolute camera positions between highend and low-cost L1 only (ambig. float) GNSS receivers. 


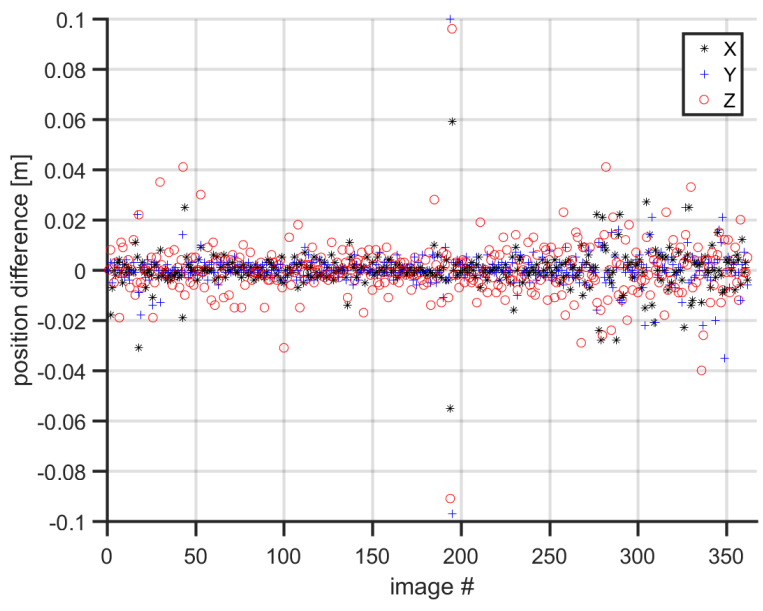

Figure 5. Differences in relative camera positions between highend and low-cost L1 only (ambig. float) GNSS receivers.

\section{MAPPING ACCURACY}

\subsection{Absolute Aerial Control}

As a next step, Pix4Dmapper Pro (Pix4D SA, 2016) was used to obtain image observations and initial attitude parameters. The reconstructed scene can be seen in Fig. 3. Bundle adjustment was carried out in TopoBun (Rehak and Skaloud, 2016) and Pix4D mapper Pro without GCPs and with self-calibrated interior orientation (IO) parameters $\left(c, x_{0}, y_{0}, K_{1}, K_{2}\right)$ in the following configurations:

I. Processing with a known lever-arm. The calibrated offset has the value of $\left.\mathbf{a}_{(} a_{x}, a_{y}, a_{z}\right)=\left[\begin{array}{lll}-433, & -31,147\end{array}\right]$ $\mathrm{mm}$. This value is a calibrated lever-arm from a ground calibration based on the pseudo-measurement technique (Ellum and El-Sheimy, 2002). This lever-arm is introduced in the adjustment as a weighted observation with $\sigma_{a_{x, y}}=1.5 \mathrm{~cm}$ and $\sigma_{a_{z}}=2 \mathrm{~cm}$.

II. Processing without a priori knowledge of the lever-arm, i.e. the initial offset is zero, its incertitude $\sigma_{a_{x}, a_{y}, a_{z}}=0.5 \mathrm{~m}$, and is estimated in BA as an additional parameter.

III. The lever-arm is not considered, i.e. it is assumed that there is no offset in camera positions.

The results of the cases I-III are summarised in Tab. 3. In general, the projects processed with camera position parameters from the
Javad receiver manifest better ground accuracy in overall. In the case the lever-arm is known, the achieved accuracy is close to 1 pixel in position and height for the Javad, and 1.5-2 pixels for the U-Blox considering the average GSD of $4 \mathrm{~cm}$.

The processing II demonstrated the ability of the BA to resolve initially unknown lever-arm, but not better than $5 \mathrm{~cm}$ along camera's x-axis. Indeed, this could be a typical mapping scenario for consumer drones to which a GNSS receiver is added, and a lever-arm between a camera and an antenna is not known. The differences between the processing I and II are significant mainly in $\mathrm{X}$ and $\mathrm{Z}$ coordinates. This is due to the unconstrained leverarm. The system is over-parametrised and estimated parameters are highly correlated, particularly the $Z_{0}-c-a_{z}$ and $x_{0}-a_{x}$. As expected, the lever-arm is highly correlated with IO parameters and camera positions, as shown in their variations in Tab. 3. There is a significant change of the $x_{0}$ coordinate between processing I and III, i.e. with and without the lever-arm. The missing lever-arm offset is absorbed by estimated values of the principal point and camera constant, but it is not projected to the ground shift in the $\mathrm{Z}$ coordinate as it happened in the case II. Some pertinent correlation parameters are stated in Tab. 4. These are calculated during the BA project of the type II with positions from the Javad receiver.

In general, the Javad receiver provided higher accuracy of absolute positions. The resulting accuracy measured at independent ChPs lies in the case I in the level of $\sim 1$ pixel in position and height, respectively. The U-Blox receiver can deliver accuracy in the level of $\sim 2$ pixels in position and $\sim 1.5$ pixels in height without the support of GCPs. Due to the size of the lever-arm, i.e. the $a_{x}$ offset is significantly larger than $a_{z}$, the horizontal ground accuracy is more influenced than its vertical component.

\begin{tabular}{|c|c|}
\hline Parameters & Correlation $[\mathbf{0 - 1 0 0 \% ]}$ \\
\hline$X_{0}-a_{x}$ & 35 \\
\hline$Y_{0}-a_{y}$ & 36 \\
\hline$Z_{0}-a_{z}$ & 79 \\
\hline$Z_{0}-c$ & 62 \\
\hline$x_{0}-a_{x}$ & 68 \\
\hline$y_{0}-a_{y}$ & 84 \\
\hline$c-a_{z}$ & 79 \\
\hline
\end{tabular}

Table 4. Significant correlations of a randomly selected image between the lever-arm $\left(a_{x, y, z}\right)$, projection centre $\left(X_{0}, Y_{0}, Y_{0}\right)$, principal point $\left(x_{0}, y_{0}\right)$, and camera constant $c$.

\subsection{Relative Aerial Control}

Relative observations were calculated from both sets of camera absolute positions. In order to orient the network, at least one GCP must be added. In practice, this can be, e.g. the base station

\begin{tabular}{|c|c|c|c|c|c|c|c|c|c|c|c|c|c|c|}
\hline \multirow{3}{*}{$\mathbf{R x}$} & \multirow{3}{*}{\multicolumn{2}{|c|}{ Test }} & \multicolumn{6}{|c|}{ Accuracy } & \multirow{2}{*}{\multicolumn{3}{|c|}{$\begin{array}{c}\text { IO } \\
{[\mathrm{mm}]}\end{array}$}} & \multirow{2}{*}{\multicolumn{3}{|c|}{$\begin{array}{c}\text { Lever-arm } \\
{[\mathrm{mm}]}\end{array}$}} \\
\hline & & & \multicolumn{3}{|c|}{ Mean ChP [mm] } & \multicolumn{3}{|c|}{ RMS ChP [mm] } & & & & & & \\
\hline & & & $\mathrm{X}$ & $\mathrm{Y}$ & $\mathrm{Z}$ & $\mathrm{X}$ & $\mathrm{Y}$ & $\mathrm{Z}$ & $\mathrm{c}$ & $x_{0}$ & $y_{0}$ & $a_{x}$ & $a_{y}$ & $a_{z}$ \\
\hline \multirow{4}{*}{ Javad } & I. & TPB: known lever-arm & -1 & 14 & 31 & 42 & 27 & 49 & 15.8315 & -0.0069 & 0.0187 & -479 & -19 & 142 \\
\hline & II. & TPB: unknown lever-arm & -1 & 14 & 93 & 41 & 27 & 100 & 15.8351 & -0.0027 & 0.0181 & -528 & -1 & 59 \\
\hline & W & TPB: no lever-arm & -1 & 15 & 107 & 46 & 36 & 115 & 15.8386 & -0.0635 & 0.0167 & - & - & - \\
\hline & III. & Pix4D: no lever-arm & 0 & -19 & -75 & 43 & 35 & 90 & 15.8421 & 0.0382 & 0.0180 & - & - & - \\
\hline \multirow{4}{*}{ U-Blox } & I. & TPB: known lever-arm & 46 & 34 & -16 & 64 & 42 & 46 & 15.8376 & -0.0050 & 0.0191 & -487 & -19 & 123 \\
\hline & II. & TPB: unknown lever-arm & 46 & 34 & 193 & 63 & 42 & 188 & 15.8491 & 0.0002 & 0.0200 & -535 & -30 & -174 \\
\hline & & TPB: no lever-arm & 47 & 35 & 47 & 67 & 47 & 63 & 15.8440 & -0.0626 & 0.0171 & - & - & - \\
\hline & III. & Pix4D: no lever-arm & -47 & -37 & -34 & 64 & 47 & 63 & 15.8452 & 0.0373 & 0.0175 & - & - & - \\
\hline
\end{tabular}

Table 3. Mapping accuracy at $23 \mathrm{ChP}$, with an absolute aerial position control, without GCPs, and with absolute aerial positions. The acronym TPB states for the TopoBun. The average GSD of these ISO projects is $4 \mathrm{~cm}$. 


\begin{tabular}{|c|c|c|c|c|c|c|c|c|c|c|c|}
\hline \multirow{3}{*}{$\mathbf{R x}$} & \multirow{3}{*}{\multicolumn{2}{|c|}{ Test }} & \multicolumn{6}{|c|}{ Accuracy } & \multirow{2}{*}{\multicolumn{3}{|c|}{$\begin{array}{c}\text { IO } \\
{[\mathrm{mm}]}\end{array}$}} \\
\hline & & & \multicolumn{3}{|c|}{ Mean ChP [mm] } & \multicolumn{3}{|c|}{ RMS ChP [mm] } & & & \\
\hline & & & $\mathrm{X}$ & $\mathrm{Y}$ & $\mathrm{Z}$ & $\mathrm{X}$ & $\mathrm{Y}$ & $\mathrm{Z}$ & $\mathrm{c}$ & $x_{0}$ & $y_{0}$ \\
\hline \multirow{2}{*}{ Javad } & I. & TPB: 1 GCP, known lever-arm & 28 & 4 & -26 & 59 & 28 & 62 & 15.8372 & -0.0063 & 0.0179 \\
\hline & I. & TPB: 4 GCPs, known lever-arm & -25 & -9 & -8 & 51 & 36 & 49 & 15.8370 & -0.0064 & 0.0180 \\
\hline \multirow{2}{*}{ U-Blox } & I. & TPB: 1 GCP, known lever-arm & 28 & 3 & -31 & 59 & 27 & 64 & 15.8390 & -0.0059 & 0.0182 \\
\hline & I. & TPB: 4 GCPs, known lever-arm & -25 & -8 & -6 & 51 & 35 & 49 & 15.8388 & -0.0059 & 0.0182 \\
\hline
\end{tabular}

Table 5. Mapping accuracy at $22 \mathrm{ChPs}$, with 1 or $4 \mathrm{GCPs}$, and with relative aerial position control.

point provided it is visible in the imagery. Thus, two scenarios are considered. Relative aerial observations with one or four, welldistributed GCPs. The processing was performed for the case I due to the assumption that a lever-arm cannot be determined well with relative position control unless the flying speed varies significantly, which was not the case. Therefore, the camera absolute positions were first corrected for the lever-arm and then differentiated between two consecutive epochs $t_{i}$ and $t_{j}$ for $d t_{t j}<10 \mathrm{~s}$, according to Eq. 2. The camera attitude values for applying lever-arm corrections were taken from BA using absolute aerial position control (within Pix4Dmapper Pro project). On the contrary, MAV platforms with short lever-arms between a camera and a GNSS antenna, such as the eBee (senseFly, 2015), can use attitude from the autopilot's IMU (Inertial Measurement Unit). For that, the autopilot's internal clock must be time-synchronised with the GNSS receiver and the camera.

The results from four adjustment projects are summarised in Tab. 5. When the relative observations of camera perspective centres replace the absolute ones and one GCP is used, the ground accuracy lies in the level of $\sim 1.5$ pixels in horizontal position and height. After adding 3 additional GCPs for the total of 4, the accuracy improved only marginally in height component by $\sim 1.5$ $\mathrm{cm}$ to $\sim 1$ pixel.

\subsection{Feasibility of L1 KAR}

Although Kinematic Ambiguity Resolution (KAR) with singlefrequency phase observations is possible, the reliability of the obtained solution is rather low as it depends not only on the quality of the obtained signal, but it requires relatively long tracking continuity without cycle slips. For this reason, a float ambiguity solution was considered for the low-cost receiver. Indeed, the convergence of float ambiguities over the time period of 5 to 10 minutes is a good indicator that L1-only KAR may be achieved over a shorter time period. The needed time span is typically 3-5 times longer than with multi-frequency data. Considering a duration of a flight-line of $60-120 \mathrm{~s}$, we have chosen a minimum of 1.5 minutes of continuous data for the KAR. This resulted in fixed aerial positions that were compared to the reference. This comparison is depicted in Fig. 6 for all used photos. The RMS of discrepancies is $<1 \mathrm{~cm}$ horizontally and $<2 \mathrm{~cm}$ vertically. As the empirically verified noise-level of the multi-frequency positioning for the employed MAV is around $2 \mathrm{~cm}$ (Rehak and Skaloud, 2015), such differences can be considered insignificant.

These results seem encouraging, yet cannot be generalised for all platforms and conditions. The requirement on longer continuity of phase data without cycle-slips is problematic under higher dynamics. Shorter observation spans lead to lower reliability of L1 KAR and thus possibly biased-position determination due to wrong ambiguities. As long as such bias is constant between subsequent photos (i.e. several seconds), it can be effectively mitigated by employing relative aerial control as investigated in (Skaloud and Lichti, 2014), (Rehak and Skaloud, 2015), and herein.

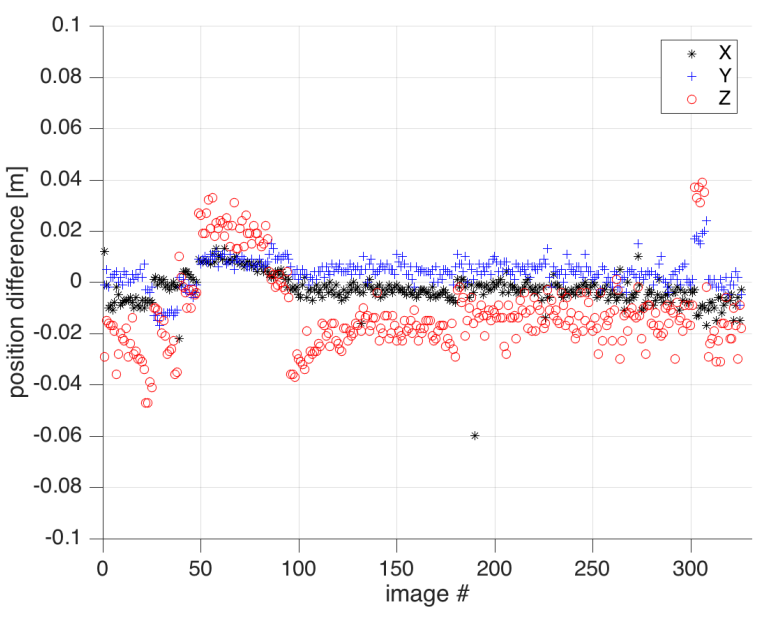

Figure 6. Differences in absolute camera positions between highend and low-cost L1 only (ambig.-fixed) GNSS receivers.

\section{CONCLUSIONS AND PERSPECTIVES}

This contribution demonstrated the potential of a low-cost GNSS receiver to deliver absolute and relative aerial observations. These observations were used in mapping configurations with and without GCPs, respectively. The results were evaluated with respect to $\mathrm{ChPs}$ accuracy, and by assessing precision values of calibrated parameters. It was shown that cm-level ground accuracy is achievable without GCPs with absolute and relative aerial observations derived from a low-cost receiver. An integration of such a receiver on a MAV platform is not trivial due to its higher sensitivity to vibrations, electromagnetic interference, and limited synchronisation possibilities. Also, the quality and/or continuity of the phase data observations on such receivers can be rapidly decreased by higher dynamics that can occur due to air turbulence, or platform's control manoeuvres. Despite that, it was empirically demonstrated that the successful L1 KAR provides practically the same precision as that of the high-end receiver. Whenever the reliability of L1 KAR is insufficient, the method of relative aerial position control provides an effective protection against positioning bias. Nevertheless, as low-cost dual (or triple) frequency receiver boards are expected to become a standard part of commercial drones in the future, the budget vs. performance ratio may lose its significance.

The article also showed the benefits of a lever-arm pre-calibration. As shown in the experiment, rather than including the lever-arm as an unconstrained parameter in the BA, it is of an advantage to let this offset be absorbed by other parameters, e.g. the interior orientation. Treating the lever-arm as an unknown parameter in the BA leads to over-parametrisation, which in turn results in higher correlations. 


\section{REFERENCES}

Aibotix GmbH, Part of Hexagon, 2016. Aibotix X6 UAV. https://www.aibotix.com/en/. [Online; accessed 13August-2016].

Benoit, L., Martin, O. and Thom, C., 2015. Low-cost GPS Sensors for deformation monitoring. GIM International 29(4), pp. 25-27.

Colomina, I., 2007. From off-line to on-line geocoding: the evolution of sensor orientation. In: D. Fritsch (ed.), Photogrammetric Week, Stuttgart, Germany, pp. 173-183.

CSG Shop, 2016. UBLOX NEO-M8T. http://www . csgshop. com/product.php?id_product $=205$. [Online; accessed 26October-2016].

Eling, C., Klingbeil, L., Wieland, M. and Kuhlmann, H., 2014. Direct georeferencing of micro-aerial vehicles - system design, system calibration and first evaluation tests. Journal of photogrammetry, remote sensing and geoinformation processing (PFG) 4, pp. 227-237.

Ellum, C. and El-Sheimy, N., 2002. Inexpensive Kinematic Attitude Determination from MEMS-Based Accelerometers and GPS-Derived Accelerations. NAVIGATION: Journal of The Institute of Navigation 49(3), pp. 117-126.

Gerke, M. and Przybilla, H.-J., 2016. Accuracy Analysis of Photogrammetric UAV Image Blocks: Influence of Onboard RTKGNSS and Cross Flight Patterns. Photogrammetrie, Fernerkundung, Geoinformation (PFG) 2016(1), pp. 17-30.

Javad GNSS Inc., 2016. Javad TRE-G3T. http: //www . javad. com/jgnss/products/oem/. [Online; accessed 14-August2016].

Li, K. and Stueckmann-Petring, J., 1992. Methods and results of combined adjustment utilizing kinematic GPS positioning and photogrammetric data. 29, pp. 213-213.

MAVinci GmbH, 2015. Sirius Pro. http://www . mavinci.com. [Online; accessed 13-August-2016].

Maxtena, 2016. Rugged L1/L2 GPS GLONASS Active Antenna. http://www.maxtena.com/products/helicore/ $\mathrm{m} 1227 \mathrm{hct}-\mathrm{a} 2-\mathrm{sma} / \mathrm{v}=1 \mathrm{ee0bf89c5d1}$. [Online; accessed 31October-2016].

Meier, L., Tanskanen, P., Heng, L., Lee, G., Fraundorfer, F. and Pollefeys, M., 2012. PIXHAWK: A micro aerial vehicle design for autonomous flight using onboard computer vision. $A u$ tonomous Robots 33(1-2), pp. 21-39.

Mongredien, C., Hide, C., Faihust, P. and Ammann, D., 2016. Centimeter positioning for UAVs and mass-market applications. GPS World.

Novatel, 2016. Waypoint Software. http://www.novatel. com/products/software/. [Online; accessed 31-October2016].

Odolinski, R. and Teunissen, P., 2016. Single-frequency, dualgnss versus dual-frequency, single-gnss: a low-cost and highgrade receivers gps-bds rtk analysis. Journal of Geodesy 90, pp. $1255-1278$.

Pix4D SA, 2016. Pix4Dmapper Pro 3.0. http://pix4d.com/. [Online; accessed 15-November-2016].

Rehak, M. and Skaloud, J., 2015. Fixed-wing micro aerial vehicle for accurate corridor mapping. In: ISPRS Annals of the Photogrammetry, Remote Sensing and Spatial Information Sciences, Vol. II-1/W4, Toronto, Canada, pp. 23-31.
Rehak, M. and Skaloud, J., 2016. Applicability of new approaches of sensor orientation to micro aerial vehicles. In: ISPRS - Annals of the Photogrammetry, Remote Sensing and Spatial Information Sciences, Vol. III-3, Prague, Czech Republic, pp. 441447.

Rehak, M., Skaloud, J. and Mabillard, R., 2013. A micro-uav with the capability of direct georeferencing. In: $U A V$-g, Rostock, Germany.

senseFly, 2015. senseFly drones and cameras. http://www . sensefly.com. [Online; accessed 13-August-2016].

Skaloud, J. Rehak, M. and Lichti, D., 2014. Mapping with MAV: Experimental study on the contribution of absolute and relative position control. In: ISPRS-The International Archives of the Photogrammetry Remote Sensing and Spatial Information Sciences, Vol. 40-3/W1, Castelldefels, Spain, pp. 123-129.

Sony, 2016. Sony NEX-5R. http://www.sony.co.uk/ support/en/product/nex-5r. [Online; accessed 14-August2016].

Stempfhuber, W. and Buchholz, M., 2011. A precise, low-cost RTK GNSS system for UAV applications. In: ISPRS - The International Archives of the Photogrammetry, Remote Sensing and Spatial Information Sciences, Zurich, Switzerland, pp. 289-293.

Survey Group, 2015. Taming a monster - largescale RTK corridor mapping in remotest Australia. https://www.sensefly.com/fileadmin/user upload/sensefly/images/web2014/applications/ senseFly-Case-Study-eBee-RTK-Talawana.pdf. [Online; accessed 12-September-2016].

U-Blox, 2016. NEO/LEA-8T. https : //www .u-blox . com/en/ product/neolea-m8t. [Online; accessed 4-September-2016]. 